\title{
A CASE REPORT OF A VARIANT HEAD OF GASTROCNEMIUS
}

\section{Ramandeep Kaur ${ }^{1}$, Anshu Sharma *2, Mahesh Kumar Sharma ${ }^{3}$.}

${ }^{1}$ Demonstrator, Department Of Anatomy, Government medical college and hospital, Chandigarh, India.

${ }^{* 2}$ Associate Professor, Department Of Anatomy, Government medical college and hospital, Chandigarh, India.

${ }^{3}$ Professor, Department Of Anatomy, Government medical college and hospital, Chandigarh, India.

\section{ABSTRACT}

The Gastrocnemius muscle is a fusiform, two-headed, two-joint muscle. The two heads of origin are lateral and medial head taking origin from medial and lateral condyles of femur. In between the two heads tibial nerve passes. Its other end forms a common tendon with the soleus muscle; this tendon is known as the tendocalcaneus or Achilles tendon which get inserted to posterior surface of calcaneum. The case was reported during routine dissection for undergraduate M.B.B.S. students in Government Medical College and Hospital, Chandigarh. The subject was 70-year-old male cadaver of North Indian origin. The accessory head was observed during the dissection of popliteal fossa. The Gastrocnemius muscle was bulky. The inferolateral and inferomedial boundary of the popliteal fossa were made by medial and lateral head of gastrocnemius. In addition to the normal medial and lateral head an accessory head of gastrocnemius was observed. The accessory head had bipennate arrangement of the muscle fibers. in the left lower limb were observed. All the heads were innervated by branches from tibial nerve. It is possible that this variant muscle could have a compressive effect on the tibial nerve and popliteal vessels, and the awareness of this may prevent its misinterpretation as a pathological structure by radiologists and surgeons.

KEY WORDS: Gastrocnemius, Soleus, Calcaneum.

Address for Correspondence: Dr. Anshu Sharma, Associate Professor, Department Of Anatomy, Government medical college and hospital, sector 32, Chandigarh, India. Mob. 9646121530

E-Mail: anshusharma_01@yahoo.co.in

Access this Article online

Quick Response code

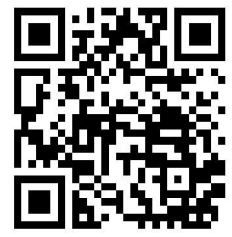

DOI: 10.16965/ijar.2018.221

Journal Information

International Journal of Anatomy and Research

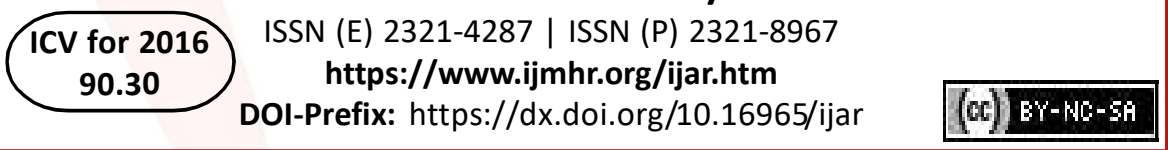

Article Information

Received: 11 Apr 2018

Peer Review: 14 Apr 2018

Revised: None
Accepted: 30 May 2018

Published (O): 05 Jul2018

Published (P): 05 Jul 2018

\section{INTRODUCTION}

The gastrocnemius muscle is a fusiform, twoheaded, two-joint muscle. It is most superficial muscle of the flexor compartment of leg and forms belly of the calf. Along with the Soleus this muscles forms triceps surae of the calf. The Gastrocnemius arises by two lateral and medial heads connected to condyles of femur by strong, flat tendons. The medial larger head is attached to depression at upper and posterior part of medial condyle behind adductor tubercle and area on popliteal surface of femur .The lateral head is attached to area on lateral surface of lateral condyle and lower part of supracondylar line [1]. The two heads come together at the inferior margin of the popliteal fossa, where they form the inferolateral and inferomedial boundaries of the popliteal fossa [2].

The tendinous attachments expand to cover posterior surface of each head with an aponeuro- 
sis, from anterior surface of which muscle fibers arise. As muscle descends, muscle fibers begin to insert into broad aponeurosis, which contracts and receives tendon of soleus on its deep surface to form Calcaneal or Achilles tendon [1].

Gastrocnemius is supplied by tibial nerve. It is supplied by its own Sural artery. These arteries are branches of popliteal artery. This muscle helps in plantar flexion [1].

It is common to have additional muscles or muscle slips in the extremities. Some of them may compress the nerves and vessels or restrict the movements, while others may enhance the muscular activity. However, a small number of them may go unnoticed [3]. Knowledge of such variant muscles becomes important for plastic surgeons while performing various reconstructive surgeries and for clinicians while managing the pain.

\section{MATERIALS AND METHODS}

During routine dissection of approximately 70-year-old male cadaver of North Indian origin, an accessory head of gastrocnemius along lateral side with bipennate arrangement of the muscle fibers in the left lower limb were observed at Department of Anatomy, Government Medical College, Sector 32, Chandigarh.

\section{CASE REPORT}

It was observed that the gastrocnemius muscle was very bulky. Along with lateral and medial heads of origin of gastrocnemius, making inferolateral and inferomedial boundary of the popliteal fossa an accessory head was seen on lateral side. The accessory head was originating from the lateral femoral condyle and adjoining part of the lateral supracondylar line as shown in the fig.1, 2 . The lateral head was originating from the lateral femoral condyle. The medial head had a single head originating from medial femoral condyle and medial supracondylar line. The tibial nerve was placed between the accessory head and medial head. All the heads of the muscle remained separate near their origin and fused to form tendocalcaneus in the lower half of leg. Another interesting observation found in this accessory head was the bipennate arrangement of fleshy fibers. All the heads were innervated by the branches of tibial nerve and supplied by popliteal vessels as shown in fig. 3. The plantaris was seen taking origin from lateral condyle of femur and was placed between the gastrocnemius and Soleus, separating the two muscles fig.2. The plantaris tendon was seen joining tendocalcaneus.

Fig. 1: Showing three heads of gastrocnemius with tendocalcenous.

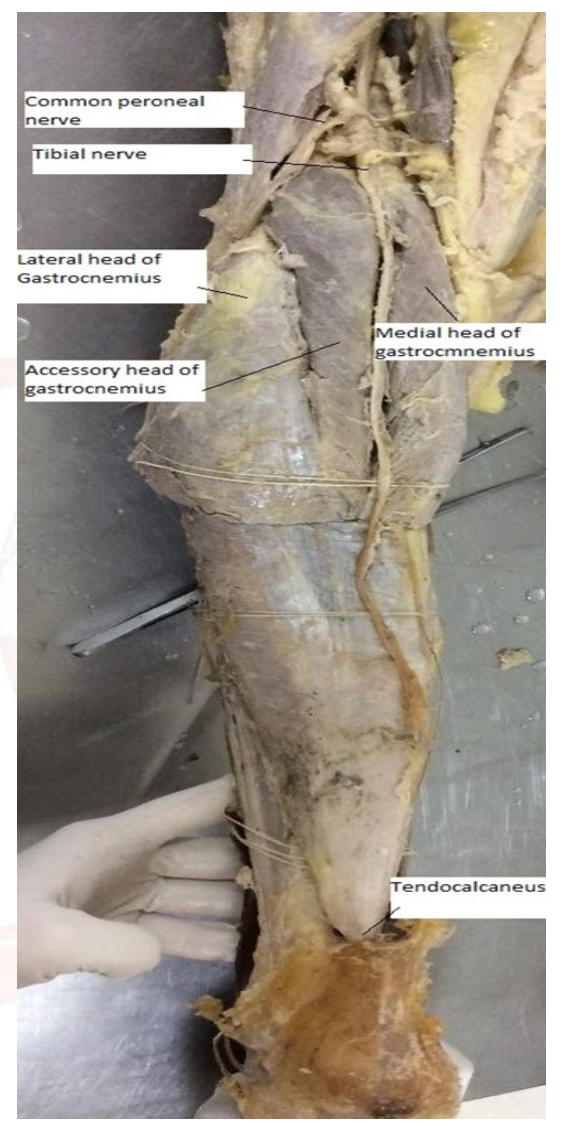

Fig. 2: Showing three heads of gastrocnemius.

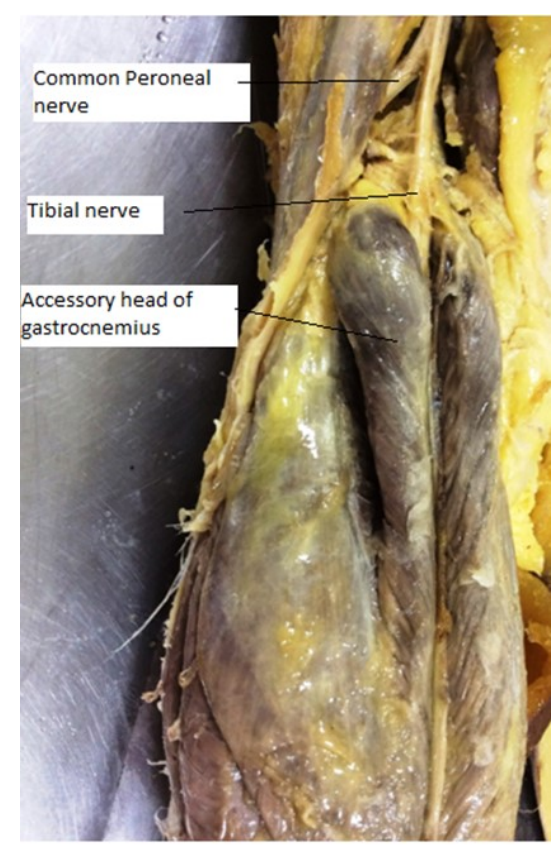


Fig. 3: Tibial nerve supplying three heads of gastrocnemius.

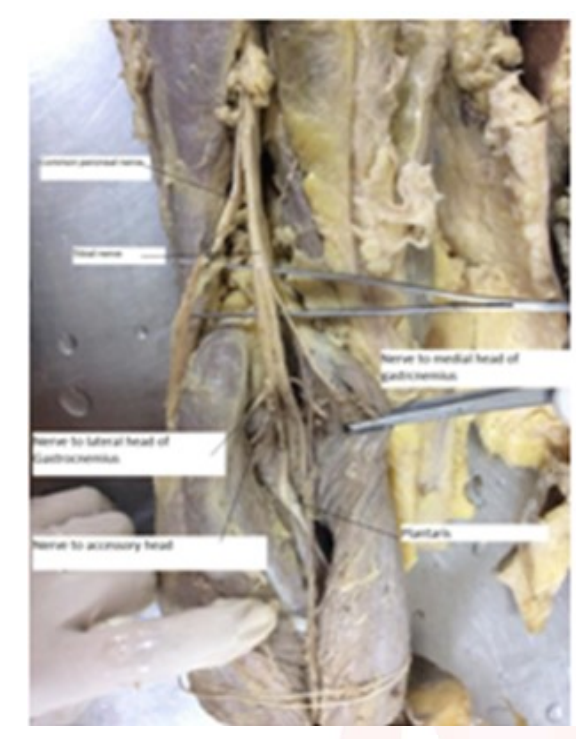

\section{DISCUSSION}

Gastrocnemius is a muscle of the calf which normally arises by two heads, from the condyles of the femur. The medial larger head is attached to the medial condyle and the lateral head is attached to lateral surface of the lateral condyle and corresponding supracondylar line. Some fibers of both the heads also arise from subjacent part of the genicular capsule. Normally, the tendinous attachments expand to cover the posterior surface of each head with an aponeurosis. From the anterior surface of this aponeurosis, the fleshy muscle fibers arise. The fleshy muscle fibers of the medial head extend lower than those of the lateral head. Below, the muscle fibers insert into a broad aponeurosis; till such point the muscular masses of two heads remain separate. The aponeurosis gradually narrows and is joined by the tendon of soleus on its deep surface to form the tendocalcaneus. Gastrocnemius is supplied by the branches of tibial nerve and popliteal artery. Presence of additional heads and other variations of the muscle is very rare. It has been mentioned that occasionally lateral head or whole muscle can be absent [3].

Kelch is the first to describe the third head. Its overall frequency ranges from $1.7 \%$ to $5.5 \%$. Phylogenetically, the gastrocnemius muscle has been considered a muscle of the fibular side of the leg. It comes from the calcaneum blastomere and follows an ascending migration towards theinferior femoral epiphysis. The third head may arise from the long head of biceps femoris muscle, linea aspera, lateral femoral epicondyle, knee joint capsule, or the fascia of leg. It may split and arise from more than one region or divide near its termination to join both heads of gastrocnemius. A rare variation where an unusual muscle that leaves the belly of semitendinosus and ends in the tendon that joins the fascia of leg is described as Tensor fasciasuralis / Ischioaponeuroticus [4].

Padmalatha et al. also reported Tensor Fascia Suralis that formed a thin tendon distally merging with the intratendinous fibers of medial and lateral heads of gastrocnemius muscle [5].

Singh et al. have reported a case where both lateral and medial heads of gastrocnemius were found to be fleshy in their entire length and were getting inserted to the calcaneum directly without forming the tendocalcaneus [6].

The third head (Caput tertium) is variation of the gastrocnemius muscle, also described as Gastrocnemius tertius seen in CT scans by Bergman [7].

Somayaji et al. have cited in their literature a case of an anomalous muscle which originated from the semimembranosus and biceps femoris muscles and then inserted into the superficial surface of the tendocalcaneus [8].

Gupta and Bhagwat found that variations in muscles are quite common and frequently seen during routine dissection of cadavers. Many or most variations are totally benign; some are errors of embryologic developmental timing or persistence of an embryologic condition. Some of these variations may seriously compromise parts of the muscular, vascular, nervous, skeletal and/or organ systems [9].

Koplas et al. carried out a prospective study of 1039 consecutive knee magnetic resonance (MR) examinations, of which 20 (1.9\%) demonstrated an anomalous third head of gastrocnemius muscle which was seen arising near the midline of the posterior distal femur, between the mid and medial aspect, and joining the medial aspect of the lateral head of the gastrocnemius. In all of these patients, the third head coursed lateral to the popliteal vessels and none coursed between the vessels [10].

The plantaris muscle itself can be considered a third head, and may join/merge with medial or 
lateral heads of gastrocnemius muscle [11]. Thus, it is advocated that the plantaris muscle is a derivative of the deeper portion of the lateral head of the gastrocnemius muscle.

Yildirim et al. have described in their report the co-existence of the bilateral gastrocnemius tertius and accessory Soleus muscles in the same cadaver. They have extensively elaborated the potential problem for structures in the popliteal fossa due to these variants. The third head joining the medial head of the gastrocnemius muscle is most commonly cited as causing clinical problems like entrapment syndromes. The characteristic signs and symptoms include leg pain, tenderness in the popliteal fossa and decreased pulsations of the distal arteries [12].

\section{CONCLUSION}

Having considered the literature available and on comparing the present case, we come to the conclusion that this case is one of the third/ accessory head of gastrocnemius muscle, originating from the popliteal surface of femur and attached distally to the junction of the medial and lateral heads of the gastrocnemius. It is possible that this variant muscle could have a compressive effect on the tibial nerve and popliteal vessels, and that the awareness of this may prevent its misinterpretation as a pathological structure by radiologists and surgeons.

\section{Conflicts of Interests: None}

\section{REFERENCES}

[1]. Williams A. Pelvic Girdle and Lower limb. In: Standring S, editor. Gray's Anatomy: The Anatomical basis of clinical practice: 39th edition. Philadelphia: Churchill Livingston Elsevier; 1949.pp 1499.
[2]. Moore K L; Agur A M, Dalley A F. Essential Clinical Anatomy: 4th edition. Baltimore: Lippincott Williams \& Wilkins; 2011. pp. 363, 365.

[3]. Standring S, Borley NR, Collins P, Crossman AR, Gatzoulis MA, Healy JC. Gray's Anatomy: The anatomical basis of clinical practice: 40th edition. London: Elsevier,Churchill Liwingstone; 2008; pp1420-21.

[4]. Bergman R A; Thompson S A, Afifi A K, Saadeh F. A. Compendium of human anatomical variation: Baltimore: Urban and Schwarzenberg, 1988; pp.7,23-4.

[5]. Padmalatha K, Prakash B S, Mamatha Y, Ramesh B. Ischioaponeuroticus/tensor fascia suralis. Int. J. Anat. Var.2011; 4:104-5.

[6]. Singh G, Agrawal VP, Basavarajappa M, Manohar M. Gastrocnemius - Achilles tendon: a human anatomical variation. Int J Biomed Adv Res. 2012; 3(08):65355.

[7]. Bergman R A, Walker CW. el-Khour G Y. The third head of gastrocnemius in CT images. Ann. Anat. 1995; 177(3):291-4.

[8]. Somayaji S N, Vincent R, Bairy K L. An anomalous muscle in the region of the popliteal fossa: case report. J. Anat. 1998; 192 ( 2):307-8.

[9]. Gupta R K. Bhagwat S S. An anomalous muscle in the region of the popliteal fossa: A case report. J. Anat. Soc. India .2006; 55(2):65-8.

[10]. Koplas M C. Grooff P. Piraino D. Recht M. Third head of the gastrocnemius: an MR imaging study based on 1039 consecutive knee examinations. Skeletal Radiol. 2009; 38(4):349-54.

[11]. Upasna \& Kumar, A. Bicipital origin of plantaris muscle - a case Report. Int. J. Anat. Var. 2011; 4:1779.

[12]. Yildirim F B. Sarikcioglu, L. Nakajima K. The coexistence of the gastrocnemius tertius and accessory soleus muscles. J. Korean Med. Sci. 2011; 26(10):1378-81.

How to cite this article:

Ramandeep Kaur, Anshu Sharma, Mahesh Kumar Sharma. A CASE REPORT OF A VARIANT HEAD OF GASTROCNEMIUS. Int J Anat Res 2018;6(3.1):5420-5423. DOI: 10.16965/ijar.2018.221 